Ann. Zootech., I978, 27 (3), 303-315.

\title{
Variations de la teneur en azote non protéique et en urée du lait à la mise à l'herbe des vaches laitières
}

\author{
B. VIGNON $(*)$, F. IAAURENT $\left({ }^{*}\right)$ et M. JOURNET $\left({ }^{* *}\right)$ \\ (*) École Nationale Supérieure d'Agronomie et des Industries Alimentaires de Nancy \\ 38 , we Sainte-Catherine, 54000 Nancy (France) \\ (**) Laboratoive de la Production laitière, \\ Centre de Recherches de Clermont-Ferrand, I.N.R.A., \\ Theix, St-Genès-Champanelle, 63110 Beaumont (Franse)
}

\section{Résumé}

L'influence de la mise à l'herbe sur les variations de la production et de la composition du lait de vache a été étudiée dans deux essais successifs.

Lorsque le régime hivernal est de bonne qualité et bien adapté aux besoins des vaches laitières, 1a mise à 1'herbe n'a qu'une très faible influence sur les quantités de lait produites et sur 1a teneur en matières azotées.

La teneur en lactose n'est pas significativement modifiée, la teneur en matières grasses augimente légèrement.

La teneur en azote non protéique (ANP) augmente très rapiđement dès la sortie au pâturage; l'écart maximum est de 25 p. roo et il se situe au cours de la I re semaine de pâturage. Cet accroissement est fugace; trois semaines après la mise à l'herbe, la teneur en ANP du lait est revenue au niveau de la période préexpérimentale.

L'urée est le principal responsable des variations enregistrées.

\section{Introduction}

Des variations importantes de la composition du lait ont généralement été observées lors du passage du régime hivernal à base de fourrages conservés à un régime d'herbe (JARRIGE, I956; ROOK et ROWLAND, I959; DEMARQUILLY et JourNET, I 962; HORST, I 963). Parmi les fractions azotées, la teneur en protéines augmente mais aussi la teneur en azote non protéique (NESENI et KöRPRICH, I948; MAIR, I95I; ROOK et ROWLAND, I959; AURIOL et JARRIGE, I962; HorST, I963; SENFT et KlobASA, r 969 ). Cette fraction azotée pourrait être à l'origine des variations constatées dans la stabilité thermique du lait ou dans le développement de 
fermentations parasites, l'azote non protéique (ANP) pouvant être une source azotée facilement utilisable par les micro-organismes contenus dans le lait.

L'élévation de la teneur en ANP à la mise à l'herbe peut être due à la consommation d'une herbe jeune. En effet, en début de premier cycle, l'herbe est riche en matière azotée (DEMARQUILLY et WEISS, I970) et en azote soluble (ZORITA, I967). L'élévation du niveau azoté de la ration accroît la teneur en ANP du sang et du lait (Rook, I,INE et RoWland, I960; Rook, I96r; AURIOL, et JARRIGE, I962; Huber et Boman, ig66; Ide, Shimbayashi et Yonemura, ig66; Prewiti et al., I97I). De même l'augmentation de la teneur en azote soluble de la ration accroît la concentration en ammoniaque du contenu du rumen et entraîne un accroissement du passage de cet ammoniaque à travers la paroi (ANNISON et LEWIS, I959; Mc DonAL,D, I 966) et une élévation de la teneur en ANP du lait (Vignon et LAURENT, I972) puisqu'il est admis que la majeure partie de l'ANP passe directement du sang à la mamelle (Corbin et WhitTiEr, ig65; MAYER, ig69). Par contre, 1'accroissement de la concentration énergátique de l'ingesta a un effet inverse (Halfpenny, Rook et Smith, I969; Gordon et Forbes, I970).

Les essais que nous avons entrepris ont consisté à préciser les fluctuations de la teneur en ANP à la mise à l'herbe en étudiant leur intensité, leur durée et la part due à l'azote uréique.

\section{Matériel et méthodes}

Deux essais ont été effectués en I969, et I970 sur le troupeau de vaches Pie Noire de 1'E.N.S.A.I.A. de Nancy.

\section{Essai $n^{0} \quad I$}

L'objectif dans cet essai a été de comparer entre eux les effets d'un régime hivernal, de la mise au pâturage et de la consommation d'herbe à l'auge. Nous avons en outre étudié les liaisons entre les variations de la composition du lait, principalement des fractions non protéiques, et les variations des apports énergétiques et azotés qui étaient connus pour les vaches alimentées à 1'auge. 'Trois lots de 6 animaux aussi homogènes que possible quant à la production laitière, au stade et au numéro de lactation ont été constitués après une période pré-expérimentale de 2 semaines pendant laquelle ils recevaient en moyenne $20 \mathrm{~kg}$ d'ensilage de maïs, I $2 \mathrm{~kg}$ de foin de prajrie permanente et $4,75 \mathrm{~kg}$ d'aliment concentré (tabl. I). Le lot A est ensuite passé au pâturage après une période de transition de r 2 jours. La période expérimentale a duré i 8 jours. Une parcelle de 2 ha a été coupée en deux sous-parcelles : l'une a été pâturée, l'autre a servi à l'afiouragement à l'auge du lot $\mathrm{B}$ dont la sortie au pâturag a a été différée de 30 jours par rapport au lot $\mathrm{A}$. Le lot $T$ utilisé comme témoin a continué de recevoir le régime hivernal mais est allé au pâturage 38 jours après le lot A. Ainsi en période post-expérimentale les animaux des 3 lots sont regroupés au pâturage.

\section{Essai $n^{\circ} 2$}

Cet essai est une répétition de $1^{\prime}$ essai $n^{0}$ I avec seulement 2 lots de 6 animaux. En période préexpérimentale ( 15 jours) les animaux des lots $\mathrm{A}$ et $\mathrm{T}$ ont reçu à volonté du foin de pré, de l'ensilage d'herbe de prairie permanente et $3 \mathrm{~kg}$ d'aliment 


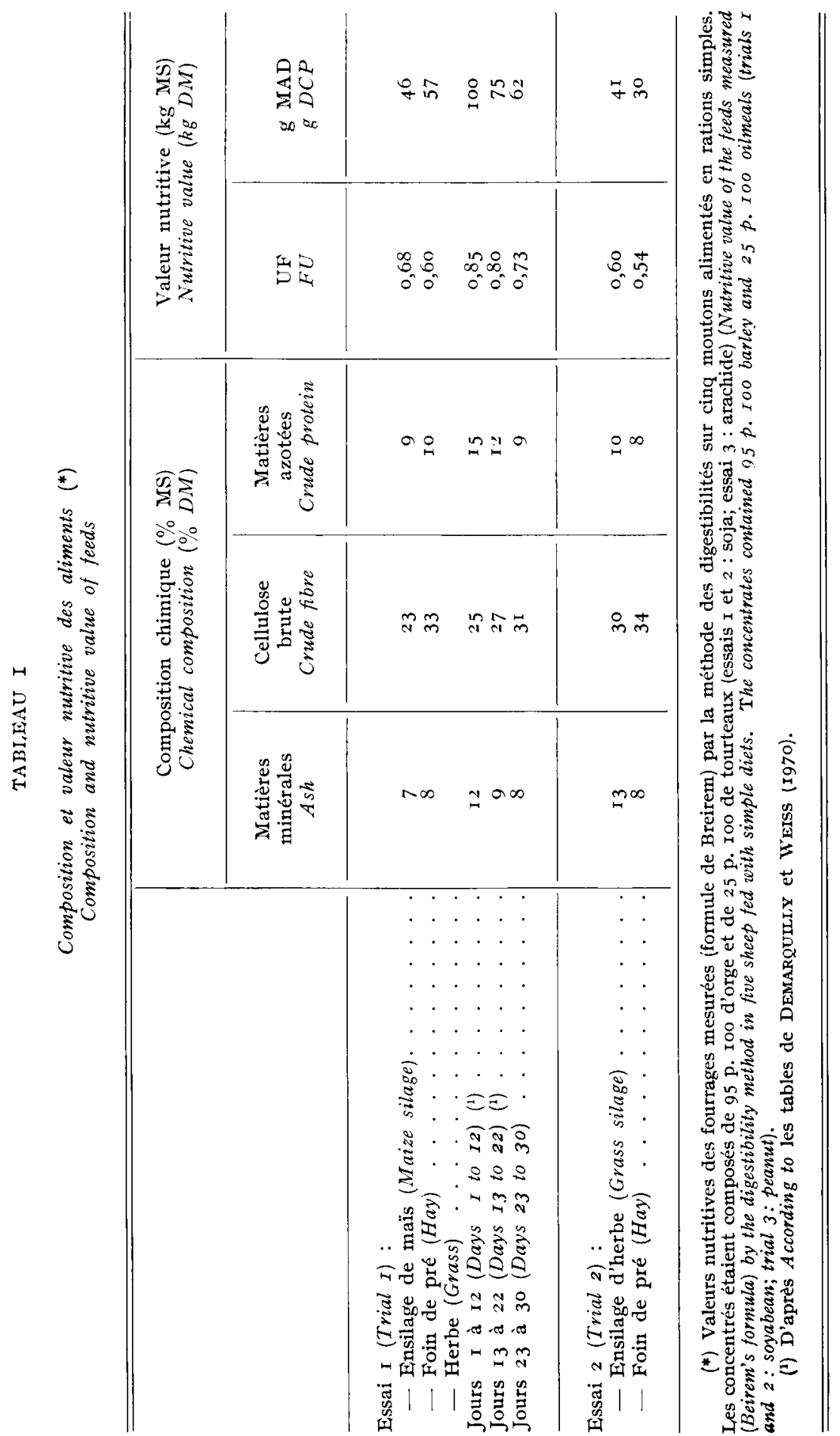


concentré (tabl. I). Les vaches du lot A sont passées progressivement (transition de 7 jours) au pâturage. La période expérimentale a duré I 2 jours puis le lot $T$ est allé au pâturage, la période de transition étant alors de 4 jours.

Les mesures de quantités d'aliments distribués et de refus et leur teneur en matière sèche ont été quotidiennes pendant les 50 jours de l'essai I. Nous avons mesuré la quantité de lait produite par vache tous les jours pendant l'essai I et en moyenne I jour sur 2 pendant l'essai 2. I)es échantillons pondérés de lait de 2 traites successives ont été prélevés en moyenne tous les 2 jours. Nous avons mesuré sur ces échantillons les teneurs en matières grasses, matières azotées totales et lactose (auto-analyseur I.R.M.A., ADDA ct al., I968). Sur le filtrat obtenu, après défécation à l'acide trichloracétique à $\mathrm{I} 2 \mathrm{p}$. Ioo de la solution finale, nous avons dosé sur tous les échantillons la teneur en azote non protéique (KJELDAHL) et la teneur en urée (mesures colorimétriques par le diacétyl monoxime, SiEsT, I968). Les données ont été regroupées par lot pour calculer les teneurs moyennes pondérées de différents composants du lait afin de disposer de résultats correspondants à des laits de mélange. Quand les lots étaient comparables en période préexpérimentale nous avons utilisé l'analyse de variance pour comparer les effets des traitements. Dans le cas contraire, nous avons procédé à une analyse de covariance des résultats individuels moyens par période en nous servant de la période préexpérimentale comme période de référence.

\section{Résultats}

\section{I. - Quantités ingérées et valeur alimentaire des rations Essai $n^{\circ}$ I (tab1. 2)}

Les vaches en régime préexpérimental ont eu un niveau d'ingestion élevé (3 $\mathrm{kg}$ de matière sèche par Ioo $\mathrm{kg}$ de poids vif). Les apports énergétiques supérieurs à $\mathrm{I} 3 \mathrm{U}$.F. /jour permettaient donc une production laitière supérieure à celle observée; les apports de matières azotées digestibles correspondaient aux besoins. Ces niveaux d'apport sont restés les mêmes au cours de la période expérimentale pour le lot témoin. Pour les vaches qui passent du régime hivernal au régime d'herbe distribuée à l'auge, le niveau des apports énergétiques est resté élevé jusqu'au I2 ${ }^{\mathbf{e}}$ jour après la mise à l'herbe, mais le niveau des apports azotés s'est accru. Après cette date, les niveaux d'apports énergétique et azoté ont diminué rapidement de près de $30 \mathrm{p}$. roo.

\section{II. - Quantités de lait produites (fig. I)}

Dans l'essai I, la mise au pâturage des animaux du lot A permet pendant les 20 premiers jours une persistance de la production laitière supérieure à celle des animaux du lot témoin. Toutefois, l'analyse de variance des résultats regroupés par période d'environ $\mathrm{I}$ semaine ne met pas en évidence de différences significatives. A partir de la $4^{\mathrm{e}}$ semaine, soit à la fin du mois de mai, par rapport aux animaux témoins, la chute de production plus importante chez les animaux consommant de l'herbe de premier cycle, aussi bien au pâturage qu'à l'auge, illustre la forte baisse de la valeur nutritive de l'herbe dès cette période. I,es animaux du lot $B$ qui reçoivent à l'étable la même herbe que celle pâturée par ceux du lot A produisent signi- 
TABLEAU 2

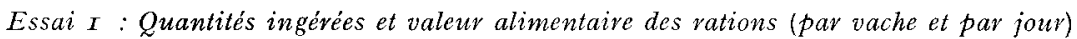
Trial $r$ : Feed intake and nutritive value of feeds (per cow and per day)

\begin{tabular}{|c|c|c|c|c|c|c|c|}
\hline & \multirow{2}{*}{ Lot } & \multicolumn{6}{|c|}{$\begin{array}{l}\text { Jours par rapport à la mise à l'herbe } \\
\text { Days before or after day of grazing }\end{array}$} \\
\hline & & $\begin{array}{l}\text { - I } 5 \\
\mathrm{a}-\mathrm{I}\end{array}$ & I $\grave{\mathrm{a}} 6$ & 7 à I $2^{7}$ & I 3 à 20 & $2 \mathrm{I}$ a $25^{2 \mathrm{I}}$ & ${ }_{26}^{30}$ à 30 \\
\hline $\begin{array}{l}\text { Ms ingérées, } \mathrm{kg} \\
\text { Dry matter intake, } k g\end{array}$ & $\begin{array}{l}\mathrm{A} \\
\mathrm{B} \\
\mathrm{T}\end{array}$ & $\begin{array}{l}18,6 \\
17,5 \\
17,9\end{array}$ & $\begin{array}{l}\text { I } 7,8 \\
\text { I } 6,9\end{array}$ & $\begin{array}{l}\text { I } 7,4 \\
\text { I } 6,7\end{array}$ & $\begin{array}{l}\overline{12,2} \\
16,9\end{array}$ & $\begin{array}{l}\text { 1 } 3,6 \\
16,7\end{array}$ & $\begin{array}{l}- \\
1_{5,4} \\
17,5\end{array}$ \\
\hline $\begin{array}{l}\text { UF ing'érées } \\
\text { FU intake }\end{array}$ & $\begin{array}{l}\mathrm{A} \\
\mathrm{B} \\
\mathrm{T}\end{array}$ & $\begin{array}{l}\text { I } 3,8 \\
\text { I } 3, \text { I } \\
\text { I } 3,4\end{array}$ & $\begin{array}{l}- \\
\text { I } 3,5 \\
\text { I } 2,8\end{array}$ & $\begin{array}{l}\text { 工 } 3,9 \\
\text { I } 2,7\end{array}$ & $\begin{array}{l}\text { т } \\
\text { г } 2,3\end{array}$ & $\begin{array}{l}- \\
\text { I } 0,6 \\
\text { I } 2,7\end{array}$ & $\begin{array}{l}\overline{\text { I I }, 8} \\
\text { I } 3,2\end{array}$ \\
\hline $\begin{array}{l}\text { MAD ingérées, } g\left({ }^{*}\right) \\
\qquad D C P \text { intake, } g\end{array}$ & $\begin{array}{l}A \\
B \\
T\end{array}$ & $\begin{array}{l}\text { I } 465 \\
\text { I } 420 \\
\text { I } 425\end{array}$ & $\begin{array}{l}\text { I } \overline{54^{\circ}} \\
\text { I } 37^{\circ}\end{array}$ & $\begin{array}{l}-\overline{ } \\
\text { I } 590 \\
\text { I } 37^{\circ}\end{array}$ & $\begin{array}{ll} & - \\
\text { I } 095 \\
\text { I } 380\end{array}$ & $\begin{array}{ll} & - \\
1 & 05^{\circ} \\
1 & 370\end{array}$ & $\begin{array}{l}\text { I } \overline{\text { I } 20} \\
\text { I } 400\end{array}$ \\
\hline
\end{tabular}

1*) Obtenues en multipliant les quantités đe matière sèche par les valeurs alimentaires moyennes (Obtained by multiplying the dry matter intake by the mean nutritive values of feeds).

ficativement ( $P=0,0 I$ ) moins de lait que celles des lots $A$ et $T$ pendant les périodes 7 à $\mathrm{I} 2$ jours et 20 à 25 jours après la mise à 1'herbe. Ces périodes correspondent à la suppression de la distribution de l'ensilage de maïs et du foin et à la réduction de 1'apport d'aliment concentré qui entraînent une baisse importante de la quantité de matière sèche ingérée, car le remplacement des autres constituants de la ration par l'herbe ne se fait que progressivement. En mettant les animaux du lot témoin au pâturage, on constate (jour $3 \mathrm{r}$ et suivants) qu'ils réduisent leur production jusqu'à un niveau identique à celui des deux autres lots.

L'essai 2 montre un effet favorable sur la production de lait aussi bien de la mise à l'herbe normale que de la mise à l'herbe différée.

\section{III. - Composition du lait}

a) Lactose, matières grasses et matières azotées

La mise à l'herbe a des effets différents sur les principaux composants du lait. La teneur en lactose n'est modifiée significativement par les différents traitements que dans l'essai I. Les teneurs en matières grasses augmentent légèrement et de façon significative.

L,es teneurs en matières azotées totales augmentent de façon significative dans le $2^{\mathrm{e}}$ essai à partir de la $2^{\mathrm{e}}$ semaine de pâturage. Ceci tend à montrer que le niveau d'apport alimentaire à l'herbe est plus important ou que les produits termi- 


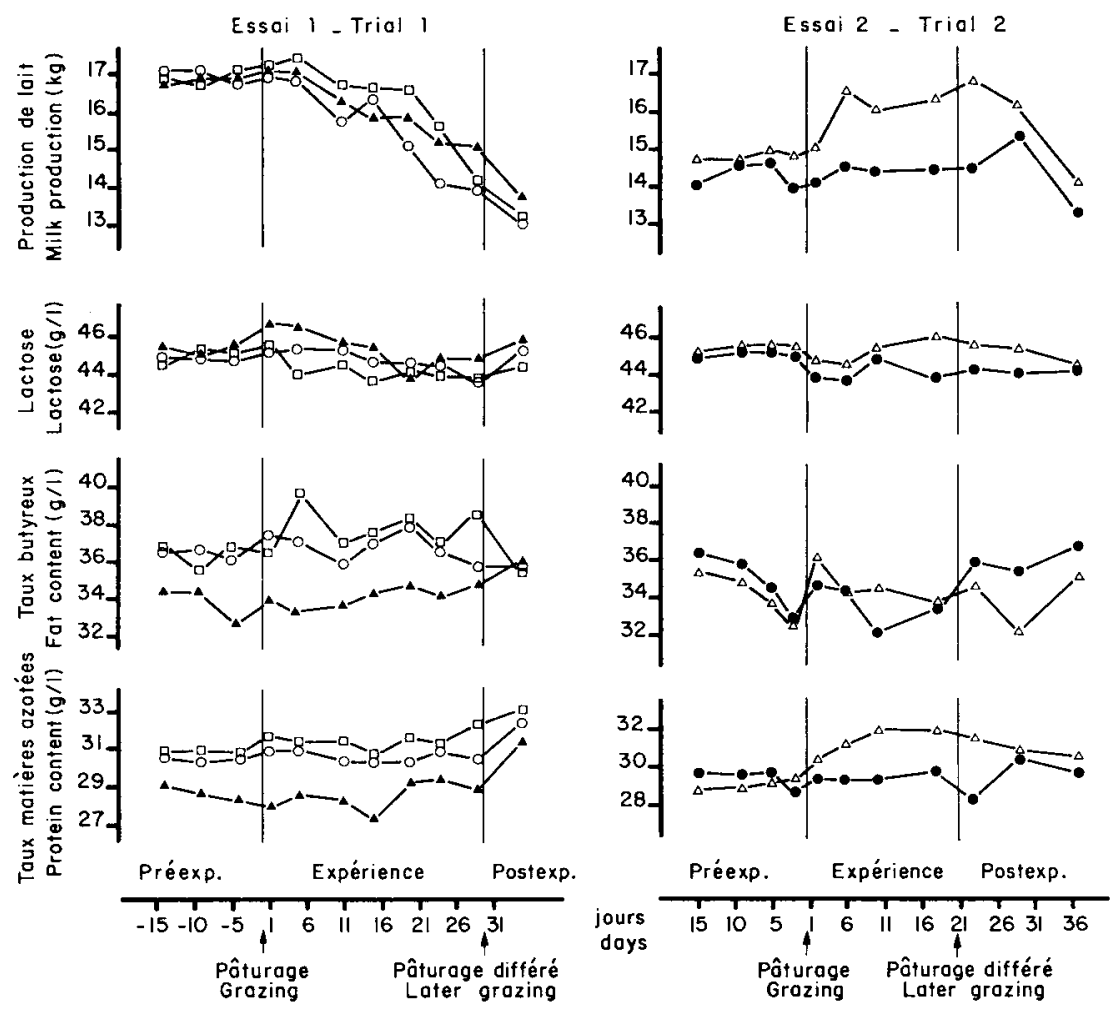

FIG. 1. - Évolution de la production de lait et de sa composition (taux moyens pondérés par lot par 3 jours).

Milk production and milk composition.
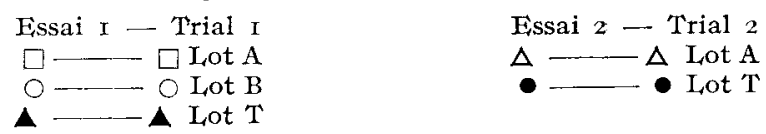

naux de la digestion sont alors plus favorables à la production de lait que dans 1e cas du régime hivernal antérieur; cet effet favorable porte autant sur les quantités de lait produites que sur le taux azoté.

b) Azote non protéique (fig. 2)

Dans l'essai I, le taux moyen pondéré d'azote non protéique du lait produit par les vaches des 3 lots n'est pas significativement différent durant les I5 jours de la période pré-expérimentale (lorsque tous les animaux reçoivent le même régime et ingèrent des quantités de matière sèche, d'énergie (U.F.) et de matières azotées digestibles équivalentes). Pour l'ensemble des animaux le taux moyen pondéré est de $258 \mathrm{mg}$ par litre. Pendant la période expérimentale, le taux d'ANP des animaux du lot témoin varie peu et les fluctuations peuvent être imputables à des facteurs aléatoires. La sortie au pâturage des vaches du lot $\mathrm{A}$ entraîne un accroissement très rapide de la teneur en ANP du lait. Le taux maximum $(325 \mathrm{mg} / \mathrm{l})$ est atteint le $4^{\mathrm{e}}$ jour; il est alors supérieur de $25 \mathrm{p}$. Ioo à celui du lait du lot témoin. 

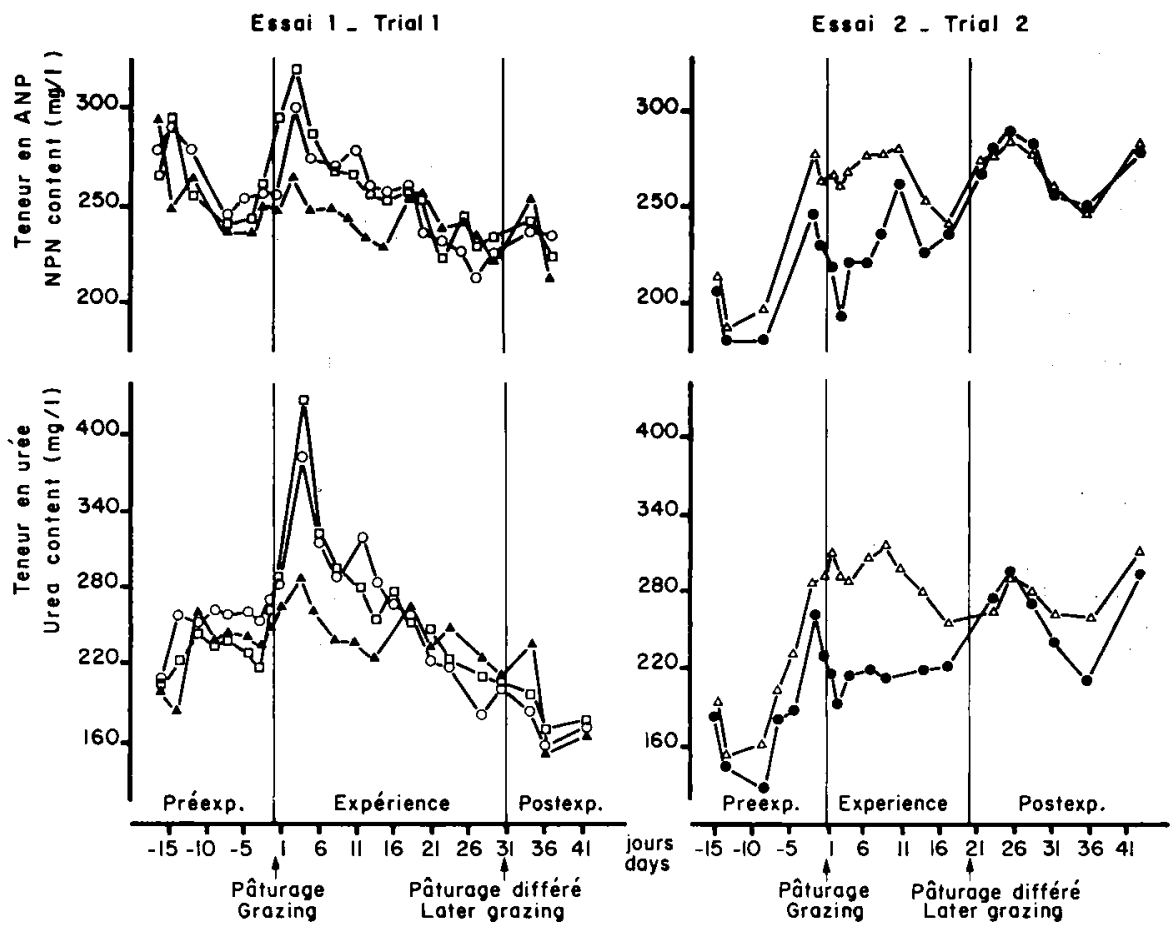

FIG. 2. - Évolution des teneurs on ANP et en urée du lait (taux moyens pondérés par lot). NPN and urea contents of milk.
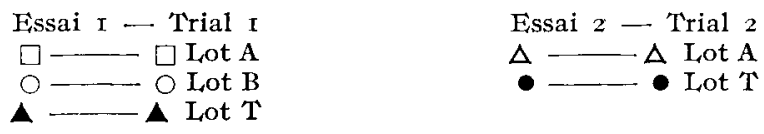

En moyenne pour les 6 premiers jours de la mise à l'herbe, le taux d'ANP augmente de I4 p. I00. L'augmentation est relativement fugace puisqu'au $20^{e}$ jour la teneur en ANP revient à sa valeur initiale. La teneur en ANP du lait des vaches du lot B consommant de l'herbe distribuée à l'auge présente une évolution semblable à celle observée pour le lot au pâturage mais l'accroissement est moins important (augmentation de l'ordre de 15 p. IOo au $4^{\mathrm{e}}$ jour). Pendant la période de transition (jour o à +6 ) les différences sont hautement significatives entre les lots $\mathrm{A}$ et $\mathrm{B}$, et le lot témoin. L'effet " jour " dans la période, qui est significatif dans les lots A et $B$, rend compte des variations importantes enregistrées entre les valeurs journalières même pour chaque lot pris isolément. Entre le $20^{\mathrm{e}}$ et le $30^{\mathrm{e}}$ jour les différences de teneur en ANP entre les lots ne sont plus significatives et la sortie différée des animaux du lot T ne provoque pas d'augmentation de la teneur en ANP tant que les animaux pâturent l'herbe de premier cycle.

Dans l'essai 2 le taux moyen pondéré d'ANP n'est pas significativement différent entre les deux lots avant la mise à l'herbe; il le devient dès la sortie du lot $\mathrm{A}$ au pâturage $(\mathrm{P}=0, \mathrm{or})$. La différence n'est plus significative après la mise à l'herbe différée du lot témoin. Les animaux des deux lots ont alors un taux d'ANP 
élevé quii est supérieur de $25 \mathrm{p}$. Ioo à celui observé pendant le régime hivernal pré-expérimental.

Les variations de la teneur en ANP du lait ne sont pas liées à celles de sa teneur en protéines. En effet, les corrélations simples, calculées dans chacun des lots sur l'ensemble de la période expérimentale ne mettent pas en évidence de liaisons significatives entre ces deux catégories de constituants.

Dans les deux essais le passage à l'herbe des animaux des lots A provoque une augmentation immédiate du rapport ANP/azote total (de 5,2 à $6 \mathrm{p}$. roo dans l'essai $\mathrm{r}$ et de 4,9 à $5,6 \mathrm{p}$. Ioo dans l'essai 2 soit une variation de $13 \mathrm{p}$. Ioo dans les deux cas). Mais ces rapports reviennent rapidement à leurs valeurs initiales (dès le $8^{e}$ jour) et l'amplitude des variations reste faible. Les différences observées entre lots passant à l'herbe et lots témoins ne sont pas significatives.

c) Urée du lait (fig. 2)

Dans l'essai I le taux moyen pondéré d'urée qui est de $239 \mathrm{mg}$ par litre pour l'ensemble des animaux augmente rapidement pour ceux mis au pâturage $(57 \mathrm{p}$. 100 au $4^{\mathrm{e}}$ jour et 27 p. Ioo sur la moyenne des 6 premiers jours). Exprimées en azote

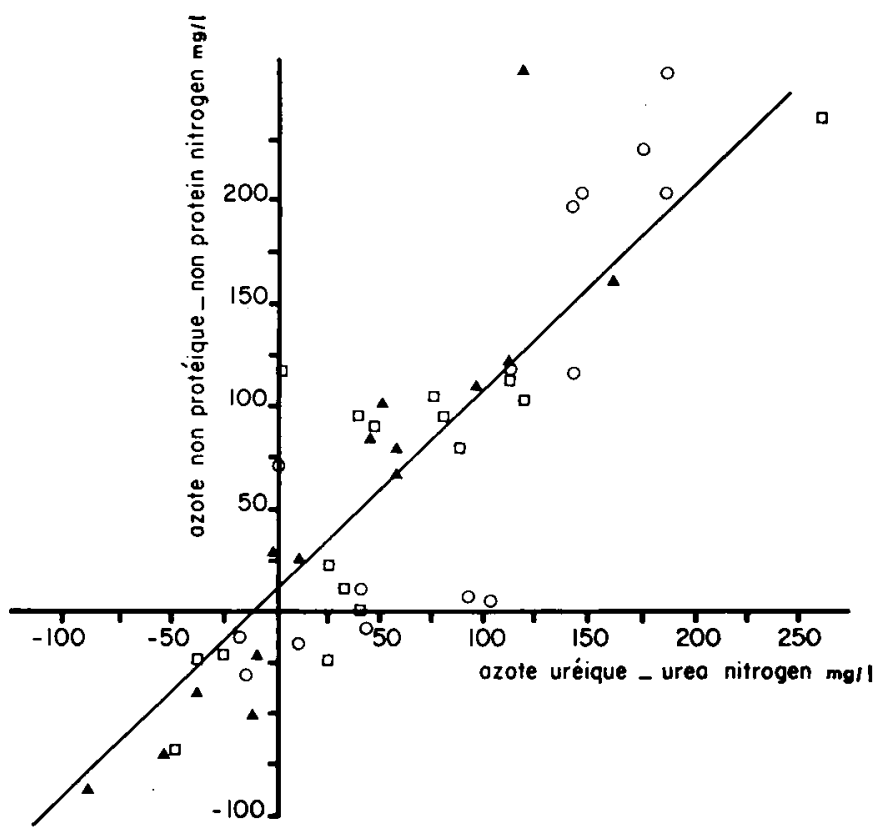

FIG. 3. - Variations des accroissements de l'azote non protéique en jonction des accroissements de l'azote uréique $\left({ }^{*}\right)$. Variations of non protein nitrogen increase according to urea nitrogen increase $\left(^{*}\right)$.

$\square$ Lot A - L ot T, essai (trial) $\mathbf{n}^{\circ} \mathbf{I}$.

$\Delta$ Lot $\mathrm{B}$ - Lot $\mathrm{T}$, essai (trial) $\mathrm{n}^{\circ} \mathrm{I}$.

O Lot A - Lot T, essai (trial) $n^{0} z$.

$\left(^{*}\right)$ En ordonnée sont portées les différences à une même date du taux d'azote non protéique entre un lot d'animaux consommant de l'herbe et le lot témoin, en abscisse les différences du taux d'azote uréique (Ordinate: NPN differences at the same date, beticen a group of grazing animals and a control group abscissa : differences in the urea nitrogen level). 
uréique, ces variations sont d'amplitude égale à celles de la teneur en ANP, ce qui montre que celles-ci sont dues presque entièrement à celles de l'urée. Pour les animaux recevant l'herbe à l'auge l'accroissement de la teneur en urée, comme pour l'ANP, est plus faible que celui des vaches au pâturage $\left(25 \mathrm{p}\right.$. roo le $4^{\mathrm{e}}$ jour et I5 p. Ioo sur la moyenne des 6 premiers jours). L'analyse de variance met en évidence la très grande variation journalière interne à chacun des lots A et B les 6 premiers jours. A partir du $20^{\mathrm{e}}$ jour il n'y a plus de différence significative entre les lots.

Dans l'essai 2, comme l'année précédente, la mise à l'herbe provoque une augmentation importante de la teneur en urée du lait parallèle à celle de 1'ANP. La différence entre les lots devient hautement significative pendant la période I à 6 jours. Entre le $8^{\mathrm{e}}$ et le $20^{\mathbf{e}}$ jour les différences ne sont plus significatives et la mise à l'herbe différée du lot témoin n'a pas d'effet sur la teneur en urée.

La part importante de l'urée et sa responsabilité dans l'accroissement de 1'ANP est confirmée par le calcul des coefficients de corrélation entre urée et ANP (intra-lot sur l'ensemble de la période expérimentale) $\left(r_{A}=0,96 ; r_{B}=0,98\right.$; $r_{\mathrm{T}}=0,80$; essai I). Les coefficients les plus élevés sont observés chez les animaux consommant de 1 'herbe.

En période préexpérimentale, pour les lots $\mathrm{A}$ dans les 2 essais, la part de l'azote uréique dans l'azote total est respectivement de 42 et 46 p. roo. En moyenne, pendant la première semaine de mise à 1'herbe, elle passe à 55 et $52 \mathrm{p}$. Ioo.

Calculé sur l'ensemble des résultats des périodes expérimentales des essais I et 2 ( 45 couples de valeurs) le coefficient de corrélation entre les accroissements de l'ANP (Y) (lait des animaux consommant de 1'herbe par rapport aux animaux témoins) et les accroissements de l'azote uréique $(x)$ est égal à 0,895 . La droite de régression de ces accroissements a pour équation : $\mathrm{Y} \operatorname{mg} / 1=0,999 \times+\mathrm{ro,7}$ (fig. 3). L'augmentation de la teneur en urée permet d'expliquer la rapidité et le caractère fugace des modifications de la composition de l'ANP.

\section{Discussion}

Les résultats de l'essai I montrent qu'avec un régime hivernal de bonne qualité et bien adapté aux besoins des vaches laitières la mise à l'herbe n'a qu'une très faible influence sur les quantités de lait produites. Lorsque le régime hivernal est moins riche (essai 2) le passage à l'herbe améliorant les apports énergétiques et azotés entraîne une augmentation de la production de lait. Des mises à l'herbe tardives sur de l'herbe âgée, donc de moins bonne valeur nutritive, ne provoquent plus ces effets favorables.

Les variations de la teneur en lactose sont d'amplitude faible comme l'étaient celles observées par Rook et Rowland (I959), Horst (I963), Vanschoubrok K (I963). La teneur en matières grasses augmente légèrement comme l'avaient rapporté précédemment JARrige et Journe't (I959), AUriol, et JARrige (I96z), DEMARquiliy et Journet (I962), VANSchoubrokK (I963), DECAEN et Ghadaki (1970). Dans l'essai 2 les teneurs en matières azotées totales augmentent de façon significative à partir de la deuxième semaine de pâturage. Ces résultats peuvent être rapprochés de ceux de JARRIGE (I956), de DEMARQUILly et JOURNET (I962) et de WAIte, CASTLE et WATSON (I959) qui mettaient en évidence un accroissement progressif de la teneur en matière azotée du lait jusqu'à la $3^{\text {e }}$ semaine suivant la mise à l'herbe. 
Le passage d'un régime hivernal à un régime d'herbe, que ce soit au pâturage ou à l'auge, provoque une augmentation rapide mais temporaire de la teneur du lait en azote non protéique avec un effet d'autant plus marqué que l'herbe consommée est plus jeune. La mise à 1 'herbe différée, lors du premier essai sur un pâturage d'herbe de $\mathrm{I}^{\mathrm{er}}$ cycle âgée donc moins riche en azote, ne modifie pas le taux d'ANP du lait. Par contre, la mise à l'herbe différée dans le $2^{\text {e }}$ essai sur des repousses de $2^{\mathrm{e}}$ cycle, accroît le taux d'ANP jusqu'au niveau de celui des animaux se trouvant déjà au pâturage. Nos résultats confirment ceux de NESENI et KöRPRICH (I948), de Rook et Rowland (I959) et de SENFT et KLOBASA (I969).

Les variations de la teneur en ANP du lait ne correspondent pas à des fluctuations identiques de sa teneur en matières azotées car la mise à l'herbe n'a qu'un effet limité sur la teneur en azote total du lait. Les liaisons entre taux d'ANP (ou taux d'urée) et taux d'azote total du lait, aussi bien chez la vache que chez la chèvre, ne sont pas significatives sauf en début de lactation où la teneur en azote total du lait diminue en même temps que la teneur en ANP. Après les premières semaines de la lactation les bilans énergétiques et azotés sont souvent positifs et c'est 1'excédent d'azote alimentaire qui influe sur la teneur en ANP (VIGNoN, I976).

Les modifications du taux d'ANP sont presque entièrement dues à celles de l'urée. En effet, les taux d'urée ont varié parallèlement aux taux d'ANP et 1'amplitude des variations de ces 2 fractions lorsqu'on les exprime en azote est identique.

Cet accroissement du taux d'urée du lait est consécutif à l'ingestion en grande quantité d'une herbe jeune. L'herbe en début de végétation a une teneur élevée en MAT (20 p. Ioo fin avril) et sa diminution est assez rapide (I I p. Ioo fin mai, I3 à I4 p. IOo pour les repousses; Morhain et al., I975). En début d'expérience les animaux du lot $B$ recevant 1'herbe à l'auge absorbent plus de MAD (200 g) que ceux du lot témoin dont la ration est calculée pour couvrir les besoins sur la base de $60 \mathrm{~g}$ de $\mathrm{MAD} / \mathrm{kg}$ de lait produit. Cette suralimentation azotée pourrait induire un accroissement de l'élimination d'azote non protéique dans le lait. Deux processus différents peuvent être impliqués. D'une part, l'augmentation de la quantité d'azote ingérée accroît la teneur en constituants azotés du rumen (surtout la fraction fermentescible), et consécutivement la teneur en ammoniaque qui passe dans le sang et qui est éliminée sous forme d'urée essentiellement dans l'urine mais aussi dans le lait. D'autre part, l'augmentation des quantités d'azote et d'énergie ingérées accroît certainement la quantité de protéines digestibles dans l'intestin d'origine alimentaire et microbienne. Champrepon, PION et Journet (I970) ont montré que l'aminoacidémie libre des vaches laitières augmente lorsqu'elles consomment de l'herbe par rapport à des régimes à base d'ensilage de maïs et d'aliment concentré. Les nutriments azotés excédentaires aux besoins sont utilisés par les tissus à des fins énergétiques, ce qui fait croître la concentration des déchets azotés. Les accroissements d'énergie et d'acides aminés disponibles lors de la mise à l'herbe pour la sécrétion de lait ne se traduisent pas toujours par une augmentation sensible de la production laitière (essai I), le niveau des apports étant déjà suffisant durant la période hivernale antérieute chez ces animauxà potentiel laitier moyen.

En plus de l'apport azoté total de la ration à la mise à l'herbe il est probable qu'intervienne la part de l'azote fermentescible dans ces matières azotées. En effet, 1'influence de la nature de l'azote alimentaire sur la teneur en ANP et en urée du lait a été mise en évidence lorsqu'on substitue l'urée à une partie des protéines alimentaires. Cette substitution dans des rations riches en azote fait augmenter au moins pendant la période d'adaptation à la nouvelle ration les concentrations en ANP et en urée du lait (VIGNON et LAURENT, I972). Inversement, l'utilisation 
de tourteaux traités au formol en remplacement de tourteaux normaux permet d'obtenir des laits moins riches en ANP (VIGNON, I976) et en urée (VERITE et JOURNET, I977).

Pour ZORITA (I967) la proportion d'ANP dans l'herbe varie de Io à 20 p. Ioo. LEFEBVRE et JOLIET (I967) montrent pour du dactyle qu'elle est plus importante au printemps qu'en été; Nowakowski et Cunningham (Ig66) indiquent pour du ray-grass d'Italie, que la part de l'azote non protéique décroit avec l'augmentation de la durée d'éclairement. La richesse en azote soluble de l'herbe jeune de printemps accroît la concentration en ammoniaque du contenu du rumen favorisant sa diffusion vers le sang. Toutefois, cette diffusion est probablement freinée par la présence simultanée de glucides solubles qui accroissent 1'efficacité de la flore du rumen dans l'utilisation de l'azote non protéique pour la synthèse microbienne. La diminution rapide de la teneur de l'herbe en matières azotées et l'adaptation de la flore du rumen à l'accroissement de matières azotées fermentescibles expliquent le retour rapide à des teneurs en ANP et en urée du lait plus normales.

Accepté pour publication en avril 1978.

\section{Summary}

Variations in the milk level of non-protein nitrogen and urea in dairy cows when changing from winter feeding to spring grazing

The effect of changing from winter feeding to spring grazing on the variations in the production and composition of cow's milk was studied in 2 successive experiments.

When the winter fodder was of good quality and well adapted to the needs of the milk cows spring grazing had only a very small effect on the quantities of milk produced and on the level of protein in the milk.

The level of lactose was not significantly affected, the level of fat slightly increased.

The level of non-protein nitrogen (NPN) increased very rapidly when the cows were put on pasture: the maximum difference (25 p. IOo) was observed during the first week of grazing. However, this increase was temporary; 3 weeks after the beginning of the grazing period, the NPN content of the milk returned to the level determined during the preexperimental period.

Urea mainly accounted for the variations recorded.

\section{Références bibliographiques}

Adda J., Blanc-Patin E., Jecnet R., Grappin R., Mocquot G., Potjatrdieu B., Ricordeau G., I968. Essais d'utilisation de 1'Infra Red Milk Analyser. Le lait, 48, I 45-153 et $293^{-302 .}$ AnNison E. F., LEwis D., I959. Metabolism in the rumen. Methuen, London, $93 \mathrm{p}$.

Auriol, P., JARrige R., I962. Possibilités de modifier la composition du lait. F.I.L.B.A., 11, $217-250$.

Champredon C., Pion R., Journet M., i97o. Influence de la nature des matières azotées alimentaires sur l'aminoacidémie libre des vaches laitières. Ann. Biol. anim. Bioch. Biophys., 10, $517-521$.

CORBIN E. A., WhitTier F. O., 1965. In Frundamentals of dairy chemistry, p. 30. Fids B. H. WEBB and A. H. Johnson. Conn. Avipublishing co., Westport. 
DECAEN C., GHADAKI M. G., I 970. Variation de la sécrétion des acides gras des matières grasses du lait de vache à la mise à l'herbe. Ann. Zootech., 15, 399-4 I I .

Demarquiliy C., Journet M., I962. Variations de la composition du lait à la mise à l'herbe. ${ }_{16} 6^{\mathrm{e}}$ Congrès Internat. de Laiterie, 1, 33-48.

Demarquiliy C., Weiss P., r97o. Tableaux de la valeur alimentaire des fourrages. Etude $n^{\circ} 42$, S.E.I.

GORDON F. J., FORBES T. J., 1970. The associative effect of level of energy and protein intake in the dairy cow. $J$. Dairy Res., 37, 48I-49I.

Halfpenny A. F., Rook J. A. F., Smith G. H., I969. Variations with energy nutrition in the concentrations of amino acids of the blood plasma in the dairy cow. $B r . J . N u t r_{-}, 23,547-557$.

Horst M. G., I963. Influence of pasture and housing periods on the composition of bulk milk. Neth. Milk and dairy $J ., 17,162-175$.

HUBER J. T., BOMAN R. L., I 966 . Effect of grain level and protein content of the grain for grazing cows on milk composition and yield and certain blood and rumen constituents. J. Dairy Sci., 49, 395-398.

IDE Y., Shimbayashr K., YoNemURA T., I966. Effect of dietary conditions upon serum and milk urea nitrogen in cows. I. Serum and milk urea nitrogen as affected by protein intake. Jap. J. Vet. Sci., 28, 32 I-327.

JARRIGE R., 1956. Variations de la teneur en matières azotées des laits individuels. $7^{\mathrm{e}}$ Congrès Internat. de Zootech., Madrid, 5, p. I I 9.

JARRIGE R., JOURNET M., I959. Influence des facteurs alimentaires et climatiques sur la teneur en matières grasses du lait. Ann. Nutr. Alim., 13, 233-278.

KLOBASA F., I969. Die Variabilität der einzelnen Stickstoffhaltigen Milchbestandteile und ihre Abhängigkeit von verschiedenen Umweltfactoren. Tierzucht. Tierernährung, 46, $85 \mathrm{p}$.

LEFEBVRE J. M., JoLIE'T F́lisabeth, I967. Étude des variations de la composition protidique et glucidique des parties aériennes du dactyle. Ann. Agron., 18, 529-543.

Mac Donald P., Edwards R. A., Greenhalgh J. F. D., I966. Animal Nutrition, I04-I43, edit. by OLIVEk and BoyD, Edinburgh and London.

MaIr H., I95I, Beiträge zur Kenntnis der Strickstoffsubstanzen der Kuhmilch, insbesondere des Restickstoffs und seiner Bestandteile. Thesis von der Fakultät für Landwirtschaft der Technischen Hochschule München, 1 I5 p.

MAYER W., 1969. Das Kolostrum einiger Haustierenten. München. Inaugural Dissertation von dev Tierarzlichen Fakultät der L. Maximiliams Universität, $236 \mathrm{p}$.

Morilain B., Blanchart G., Jaumain J. L., Vignon B., I975. Valeur alimentaire de l'herbe de prairie permanente lorraine utilisée en vert, en ensilage et en foin et productivité de la prairie. Bull. E.N.S.A.I.A., Nancy, 17, 3-29.

NESFNI R., KORPRICH H., 1948. Der Reststickstoffgehalt in normaler Kuhmilch. Milchwissenschaft, 3, I 83-I 86 .

Nowakowski T. Z, CUnningham R. K., 1966. Nitrogen fractions and soluble carbohydrates in italian Ryegrass. II. Fffects of light intensity, form and level of nitrogen. J. Sci. Fd. Agric., 17, I 45 -I 50 .

Prewitt L. R., Kentz A. F., Lane A. (;., Cimpheli J. R., Weinman D. E., i97 I. Effect of dietary protein on blood urine and milk composition. Amer. J. Vet. Res., 32, 393-397.

RoOk J. A. F., I96I. Variations in chemical composition of the milk of the cows. Part I. Dairy Sci. Abstr., 23, 25I-258.

Rook J. A. F., I 96I. Variations in chemical composition of the milk of the cows. Part 2. Dairy Sci. Abstr., 23, 303-307.

Rook J. A. F., Rowland S. J., I959. Effect of spring grazing on the constituents of the solids not fat of milk. XI'e Intern. Dairy Congr., A, zO4-zog.

ROOK J. A. F., LINE C., ROWLAND S. J., I960. The effects of the plane of energy nutrition of the cow during the late winter feeding period on the changes in the S.N.F. content of milk during the spring grazing period. J. Dairy Res., 27, 427-433.

SENFT B., KLOBASA F., I969. Uber den Einfluss verschiedener Umweltfaktoren auf den Gehalt der Milch in Nichtproteinstickstoff. Milchwissenschaft, 24, 7 I 3-7I 7 .

Siest G., I968. Fitude de la réaction urée-diacétylmonoxine. II. Essai de divers adjuvants. Choix d'une méthode de dosage. Ann. Biol. Clin., 26, 43 I-448.

VANSchOUBRokK F. X. E. J., I963. Determination and variation of protein, fat and lactose in cow's milk and selection on these factors. Neth. milk and dairy J., 17, $12-45$. 
VERITE R., JouRneT M., I977. Utilisation des tourteaux traités au formol par les vaches laitières. II. Effets sur la production laitière du traitement des tourteaux et du niveau d'apport azoté au début de la lactation. Ann. Zootech., 26, I83-205.

Vignon B., I976. La fraction azotée non protéique du lait, importance et variations. Thèse, Nancy, $245 \mathrm{p}$.

Vignon B., LAURen'T F., 1972. Effet de la supplémentation des vaches laitières en urée sur la composition du lait. Bull. E.N.S.A.I.A., Nancy, 14, 13-22.

WAITE R., CASTLE M. E., WATSON J. N., 1959. The effect on milk composition of feeding spring grass to cows. J. Dairy Res., 26, 173-181.

ZORITA E., 1967. In Handbuch der Futtermittel, 3, 290-295, herausgegeben von Max Becker, Kurt Nehring. Verlag Paul Barey, Berlin. 